therapy. This low proportion $(9.5 \%)$ is in stark contrast to the USA where, mainly as a result of policy directives in line with the goal of TB elimination in the USA [6], 74-79\% of patients diagnosed with LTBI commence treatment $[7,8]$. In our cohort of contacts, low treatment rates for LTBI appeared to be mainly a consequence of physicians' reluctance to offer LTBI treatment rather than low patient uptake of treatment.

In conclusion, in a setting where more than half of all screened contacts were born overseas and the background incidence of TB is low, contacts of patients with TB have a significantly increased risk for TB compared to the general overseas-born population of Australia. Most contacts who develop active TB are diagnosed with $\mathrm{TB}$ at the time of the initial screening, thus efforts should be made to ensure at least one screening visit for every identified contact at risk. Few contacts received treatment for LTBI in this study setting, due to physicians' reluctance to prescribe preventive treatment.

\section{Claudia C. Dobler ${ }^{*, \#}$ and Guy B. Marks*,\# \\ *Respiratory and Environmental Epidemiology, Woolcock Institute of Medical Research and Central Clinical School, University of Sydney, Sydney, and "Dept of Respiratory Medicine, Liverpool Hospital, University of New South Wales, Sydney, Australia.}

Correspondence: C.C. Dobler, Woolcock Institute of Medical Research, PO Box M77, Missenden Road, NSW 2050, Australia. E-mail: cdobler@med.usyd.edu.au

Support Statement: C.C. Dobler is supported by a University of Sydney Postgraduate Award Scholarship. There was no other study funding. The funders of the research scholarship had no role in study design, data collection and analysis, decision to publish, or preparation of the manuscript.
Acknowledgements: We acknowledge Rose Ampon (Woolcock Institute of Medical Research, University of Sydney, Sydney, Australia) for performing the database linkage. We also acknowledge Con Tsiavos (Sydney West Area Health Service, Sydney, Australia) who wrote the script for data extraction from the CSS database. Our thanks go to Jeremy McAnulty (New South Wales Ministry of Health, Sydney, Australia), who granted access to the New South Wales TB notification database and Amanda Christensen, (New South Wales TB Program Manager; New South Wales Ministry of Health) who facilitated the data linkage.

\section{REFERENCES}

1 Targeted tuberculin testing and treatment of latent tuberculosis infection. This official statement of the American Thoracic Society was adopted by the ATS Board of Directors, July 1999. This is a Joint Statement of the American Thoracic Society (ATS) and the Centers for Disease Control and Prevention (CDC). This statement was endorsed by the Council of the Infectious Diseases Society of America. (IDSA), September 1999, and the sections of this statement as it relates to infants and children were endorsed by the American Academy of Pediatrics (AAP), August 1999. Am J Respir Crit Care Med 2000; 161: S221-S247.

2 Barry C, Waring J, Stapledon R, et al. Tuberculosis notifications in Australia, 2008 and 2009. Commun Dis Intell 2012; 36: 82-94.

3 Campbell KM, Deck D, Krupski A. Record linkage software in the public domain: a comparison of Link Plus, The Link King, and a "basic" deterministic algorithm. Health Informatics J 2008; 14: 5-15.

4 Fox GJ, Barry SE, Britton WJ, et al. Contact investigation for tuberculosis: a systematic review and meta-analysis. Eur Respir J 2013; 41: 140-156.

5 Marks GB, Bai J, Simpson SE, et al. Incidence of tuberculosis among a cohort of tuberculin-positive refugees in Australia: reappraising the estimates of risk. Am J Respir Crit Care Med 2000; 162: 1851-1854.

6 Tuberculosis elimination revisited: obstacles, opportunities, and a renewed commitment. Advisory Council for the Elimination of Tuberculosis (ACET). MMWR Recomm Rep 1999; 48: 1-13.

7 Marks SM, Taylor Z, Qualls NL, et al. Outcomes of contact investigations of infectious tuberculosis patients. Am J Respir Crit Care Med 2000; 162: 2033-2038.

8 Anger HA, Proops D, Harris TG, et al. Active case finding and prevention of tuberculosis among a cohort of contacts exposed to infectious tuberculosis cases in New York City. Clin Infect Dis 2012; 54: 1287-1295.

\title{
Using the Chartis system to selectively target a lung segment with a persistent air leak
}

\section{To the Editor:}

Persistent air leak (PAL) is an important complication of pneumothorax, especially of secondary pneumothorax. PAL increases not only the morbidity and mortality of patients, but also the length of hospital stay and costs. When a chest drain and suction fail to stop the PAL, surgical intervention is recommended according to the British Thoracic Society guidelines on pneumothorax treatment [1]. Pleurodesis is considered as an alternative treatment when surgical repair is impossible due to an underlying comorbidity. Diverse chemical and biological substances, including autologous blood [2], talc [3, 4], fibrin [5] and antibiotics [6], have demonstrated various efficiencies to treat PAL. Recently, an endobronchial valve (EBV) was successfully utilised to treat PAL [7-9]. Targeting the lung lobe or segment with an air leak is a prerequisite for stopping PAL using EBV. In the present study, we report a novel way of identifying the precise location of an air leak with a catheter-based device (Chartis system; Pulmonx Inc., Redwood City, CA, USA), which can measure airway pressure and flow.

A 49-year-old male presented to the emergency department with dyspnoea. His medical history included recurrent right-sided pneumothorax, for which he had received a video-assisted 
thoracoscopic lobectomy 3 years earlier. Chest computed tomography revealed pneumothorax on the left side. The air leak did not show any downward trend during a 45-day period after pleural tube placement. Pleurodesis with tetracycline was performed through the chest tube and proved to be futile on the PAL.

Before EBV was carried out to treat the PAL, we performed an assessment using the Chartis system to determine the exact lobe or segment with PAL. As most cases of spontaneous pneumothorax originate from the upper lobes, the left upper lobe (LUL) was selected to be assessed as a priority. After the main bronchus of the LUL was occluded by the balloon of the Chartis catheter, a negative pressure $\left(-10 \mathrm{cmH}_{2} \mathrm{O}\right)$ was exerted to the exhaust port of the thoracic drainage bottle. The patient's inspiratory pressure was overlapped with the negative pressure created by the vacuum, but the former dominated in the pressure waveform (fig. 1). However, after the catheter was inserted into the bronchus B1 and the balloon sealed the S1 segment, a significant and constant negative pressure was observed (fig. 1b). An EBV (Zephyr; Pulmonx Inc.) was deployed in bronchus B1, and the air leak decreased dramatically after the treatment. 3 days after EBV placement, the air leak stopped and the chest tube was removed.

EBV placement has evolved as an alternative pneumothorax treatment to surgical repair after the failure of chest tube placement, especially in patients not suitable for invasive interventions. Before the deployment of an EBV, when the patient was still undergoing treatment with chest tube drainage, a balloon-catheter occlusion method is used to identify the lobe and segment containing the air leak [7-9]. Lobar and segmental airways can be occluded sequentially by the balloon via the working channel of bronchoscopy. A substantial decrease of the air leak implies the occlusion of lobar or segmental airways connected to the culprit air leak. Although this method is not complicated, it was criticised for subjective judgement and non-quantitativeness. Recently, a new digital thoracic drainage system, which allows
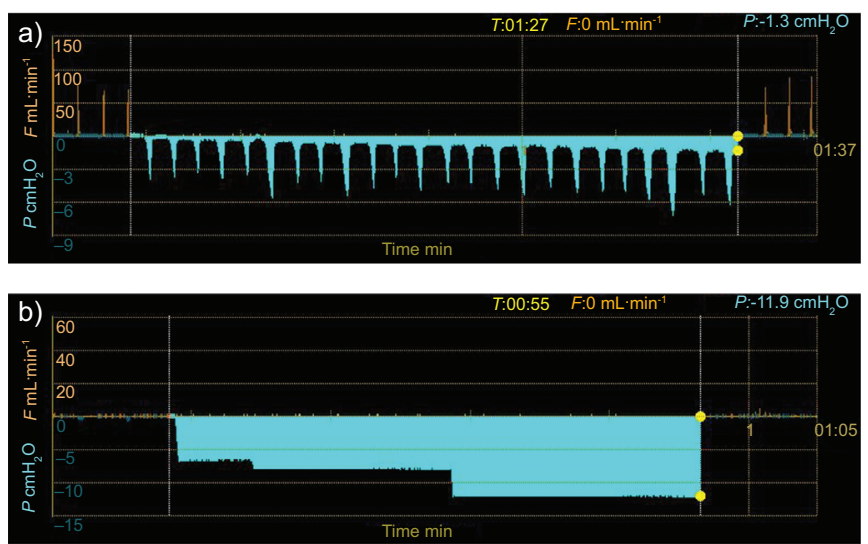

FIGURE 1. Using the Chartis system (Pulmonx Inc., Redwood City, CA, USA) to identify the lung segment with an air leak. a) When the balloon has occluded the lobar bronchus of the left upper lobe, the negative pressure generated by the vacuum was displayed as a low-level $\left(<2 \mathrm{cmH}_{2} \mathrm{O}\right)$ continuous negative pressure at both the inspiratory and expiratory phases. b) Occlusion of bronchus B1 resulted in a high-level sustained negative pressure, concealing the negative pressure of the inspiratory manoeuvre. $T$ : time; $P$ : pressure; $F$ : flow. assessment of the leakage objectively and accurately, was used to guide the EBV placement [10].

In the present study, we described how to target air leak location using the Chartis system, which was applied to assess collateral ventilation before endobronchial lung volume reduction. The Chartis system integrates a one-way valve into the console and can measure the air flow and collateral resistance simultaneously and dynamically. In the case of pneumothorax, the negative pressure can be conducted from thoracic cavity to the airways through the fistula, and finally to the console through the catheter. In this patient, when the balloon had occluded the lobar bronchus of the LUL, indicated by the disappearance of the airflow, the negative pressure created not only by inspiratory manoeuvre but also by the vacuum could be detected. The latter was manifested as a low-level $\left(<2 \mathrm{cmH}_{2} \mathrm{O}\right)$ continuous negative pressure at both the inspiratory and expiratory phases. However, occlusion of bronchus B1 resulted in a high-level sustained negative pressure concealing the negative pressure created by the inspiratory manoeuvre. The source of air leakage was localised at the apical segment of the LUL. Separation of apical segment from other parts of LUL made the negative pressure from thoracic cavity transfer directly to the Chartis console though the lung with air leak without redistribution in the whole LUL.

In conclusion, the Chartis system may be used to identify the segment of lung with an air leak and selectively guide the EBV placement. Selectively targeting one segment of lung may conserve the lung function of chronic obstructive pulmonary disease patients with limited respiratory reserve, and make EBV treatment affordable for more patients.

\section{Qing Tian, Fei Qi, Yang An, Xing-Chen Liu and Liang-an Chen \\ Dept of Respiratory Diseases, PLA General Hospital, Beijing, China.}

Correspondence: L. Chen, Dept of Respiratory Diseases, PLA General Hospital, Fuxing Road No.28, Beijing 100853, China. E-mail: chenla301@163.com

Statement of Interest: None declared.

\section{REFERENCES}

1 MacDuff A, Arnold A, Harvey J, et al. Management of spontaneous pneumothorax: British Thoracic Society pleural disease guideline 2010. Thorax 2010; 65: Suppl. 2, ii18-ii31.

2 Rinaldi S, Felton T, Bentley A. Blood pleurodesis for the medical management of pneumothorax. Thorax 2009; 64: 258-260.

3 Dubois L, Malthaner RA. Video-assisted thoracoscopic bullectomy and talc poudrage for spontaneous pneumothoraces: effect on shortterm lung function. J Thorac Cardiovasc Surg 2010; 140: 1272-1275.

4 Kilic D, Findikcioglu A, Hatipoglu A. A different application method of talc pleurodesis for the treatment of persistent air leak. ANZ J Surg 2006; 76: 754-756.

5 Finch CK, Pittman AL. Use of fibrin glue to treat a persistent pneumothorax with bronchopleural fistula. Am J Health Syst Pharm 2008; 65: 322-324. 
6 Liberman M, Muzikansky A, Wright CD, et al. Incidence and risk factors of persistent air leak after major pulmonary resection and use of chemical pleurodesis. Ann Thorac Surg 2010; 89: 891-897.

7 Travaline JM, McKenna RJ Jr, De Giacomo T, et al. Treatment of persistent air leaks using endobronchial valves. Chest 2009; 136: 355-360.

8 El-Sameed Y, Waness A, Al Shamsi I, et al. Endobronchial valves in the management of broncho-pleural and alveolo-pleural fistulae. Lung 2012; 190: 347-351.
9 Abu-Hijleh M, Blundin M. Emergency use of an endobronchial one-way valve in the management of severe air leak and massive subcutaneous emphysema. Lung 2010; 188: 253-257.

10 Dooms CA, De Leyn PR, Yserbyt J, et al. Endobronchial valves for persistent postoperative pulmonary air leak: accurate monitoring and functional implications. Respiration 2012; 84: 329-333.

DOI: 10.1183/09031936.00184712 